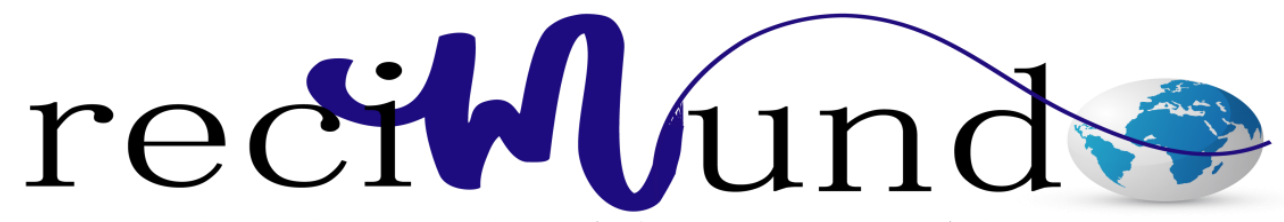

Revista Científica Mundo de la Investígación y el Conocimiento

Blanca Esthela Moscoso ${ }^{\mathrm{a}}$; Reema Azar ${ }^{\mathrm{b}}$

Influencia de la Identidad Cultural en la Percepción Ciudadana - Caso de Estudio

Revista Científica Mundo de la Investigación y el Conocimiento. Vol. 1 núm., 5, diciembre, 2017, pp. 799-813

DOI: $10.26820 /$ recimundo/1.5.2017.799-813

Editorial Saberes del Conocimiento 


\section{Influencia de la Identidad Cultural en la Percepción Ciudadana - Caso de Estudio}

Vol. 1, núm. 5., (2017)

Blanca Esthela Moscoso; Reema Azar

\section{RESUMEN}

Los Estados Unidos se dividen en gran medida entre los votantes que apoyan o condenan la Orden Ejecutiva del presidente Trump que prohíbe la entrada de refugiados a los Estados Unidos. Estas divisiones se pueden ver a lo largo de las líneas de los diferentes partidos políticos y de las demográficas de edad. Los demócratas y los votantes menores a 40 años tienden a criticar la "prohibición musulmana", mientras que los republicanos y los votantes mayores a 40 abogan por su implementación. Inclusive los sirio-estadounidenses están divididos sobre el tema, a pesar de que esta prohibición afecte directamente a sus propios familiares y amigos. La cultura de los EE. UU. parece estar dividida, mientras unos apoyan a la prohibición musulmana, otros la critican. Pese a que la libertad para todos está representada en los iconos nacionales dominantes en la identidad de los Estados Unidos, el etnocentrismo ha llevado a la xenofobia a una gran parte de la población. Este documento presenta una idea global de la división de la población de votantes de EE. UU. y el papel que la cultura ha desempeñado en la configuración de puntos de vista opuestos sobre la prohibición de refugiados.

Palabras Claves: Orden ejecutiva de Trump, prohibición musulmana, prohibición de refugiados, prohibición de inmigración, etnocentrismo. 


\title{
Influencia de la Identidad Cultural en la Percepción Ciudadana - Caso de Estudio
}

Vol. 1, núm. 5., (2017)

Blanca Esthela Moscoso; Reema Azar

\begin{abstract}
The United States is largely divided between voters who either support or condemn President Trump's Executive Order banning refugees from entrance into the U.S. These divisions can be seen along party lines and age demographics; Democrats and voters under the age of 40 tend to criticize the "Muslim ban," while Republicans and voters over the age of 40 advocate for its implementation. Even Syrian-Americans are split on the issue, despite the fact that the ban directly impacts their own family members and friends. The culture of the U.S. appears to support the ban on the one hand, while contradicting it on the other. Ethnocentricity has led to xenophobia among a large portion of the population, though national icons dominant in U.S. identity represent freedom for all. This paper will offer insight into the division of the U.S. voter population and the role that culture has played in shaping opposing views of the refugee ban.
\end{abstract}

Key Words: Trump Executive Order, Muslim ban, refugee ban, immigration ban, ethnocentrism. 


\section{Influencia de la Identidad Cultural en la Percepción Ciudadana - Caso de Estudio \\ Vol. 1, núm. 5., (2017) \\ Blanca Esthela Moscoso; Reema Azar}

\section{Introducción.}

El presidente de los Estados Unidos, Donald Trump, emitió la Orden Ejecutiva 13769 el 27 de enero del 2017, una de sus primeras acciones como el nuevo líder electo de una nación diversa y políticamente frustrada. Esta "prohibición musulmana", a la que se ha referido en gran medida, impidió indefinidamente el ingreso a los Estados Unidos a los refugiados que huían de la guerra civil siria. La prohibición estadunidense manifestó inmediatamente su indignación de que un país fundado en principios de libertad religiosa cerrara sus puertas a aquellos de necesidad extrema. Sin embargo, tales sentimientos no fueron compartidos por todos los ciudadanos; ya que la nación se dividió entre quienes abogaron por dicha prohibición y en quienes se opusieron a ella. La identidad cultural de los EE. UU. tuvo un papel importante en la formación de las opiniones de una nación dividida. Mientras la nación debatía incansablemente sobre su destino, los sentimientos de etnocentrismo conflictuaron con los símbolos de libertad e igualdad de oportunidades del país, dejando a una población altamente vulnerable_refugiados a la espera de entrar en los EE. UU.- en el limbo. Tanto el apoyo como las críticas recibidas con respecto a la Orden Ejecutiva, cuestionan los valores de una nación de contradicciones.

\section{Antecedentes}

Escenario Electoral en el 2016

En las elecciones presidenciales más recientes de Estados Unidos, muchos partidarios del candidato Donald Trump se sintieron atraídos por su uso de una retórica fundada en la ira. Múltiples estudios han demostrado (Shiv et al. 2005; A. J. Banks 2016) que la ira juega un papel poderoso en la formación de las emociones; de hecho, la ira que inicialmente no se relaciona con 


\section{Influencia de la Identidad Cultural en la Percepción Ciudadana - Caso de Estudio}

Vol. 1, núm. 5., (2017)

Blanca Esthela Moscoso; Reema Azar

grupos raciales o étnicos, puede llegar a convertirse en emociones negativas hacia estos grupos.

Por ejemplo, un entorno en el que las personas están enojadas por la economía, por la infraestructura que se desmorona o por el costo de la atención médica, podría llevar a las personas a ser chivos expiatorios de grupos étnicos y minoritarios y, por lo tanto, a oponerse a acciones gubernamentales que podrían beneficiar a estos grupos (A. Banks 2017). La ira es una emoción que indudablemente resulta en la culpa de otros; una retórica llena de enojo durante un proceso electoral es efectiva porque une a los grupos que resuenan con esta emoción, mientras que divide a los que pueden ser víctimas de la culpa.

La ira lleva a las personas a desarrollar actitudes fuertes para enfrentar a un enemigo común (Claassen 2016; Mackie, Devos, and Smith 2000), ya sea de manera violenta o no violenta. Por ejemplo, un estudio que utilizó datos de la elección nacional americana entre 1992 y 2004 concluyó que la ira no relacionada con grupos raciales o étnicos tiene un efecto sobre los "blancos" etnocéntricos para oponerse firmemente a las políticas raciales y de inmigración, moviendo a aquellos con tendencias etnocéntricas más bajas a apoyar estas políticas (A. J. Banks 2016).

\section{Identidad Cultural}

La identidad social es la autopercepción de pertenecer a otros grupos con características comunes, creencias, rasgos culturales, raíces, etc. (Hogg 2016). La identidad social es una construcción compleja que se refiere a la auto-identificación subjetiva del individuo y de cómo este se relaciona con otros grupos de distinta naturaleza y dimensiones (Roccas and Brewer 2002). La identidad cultural es uno de los lentes que usa la identidad social, enfatiza el sentido de 


\section{Influencia de la Identidad Cultural en la Percepción Ciudadana - Caso de Estudio \\ Vol. 1, núm. 5., (2017) \\ Blanca Esthela Moscoso; Reema Azar}

pertenencia debido a sus raíces culturales, su lugar de origen, su religión, etc. Sin lugar a dudas, la raza juega un papel importante en la identidad cultural porque está interrelacionada con la religión, las tradiciones y las raíces del origen (King 2004).

Estas relaciones interrelacionadas pueden ser la causa de la tensión entre los distintos miembros de un grupo. Por ejemplo, en un estudio realizado en Canadá, los grupos musulmanes identificaron las tensiones de identidad cultural entre la definición del "derecho a hablar" en su tierra actual y algunas "creencias religiosas profundas" de sus tradiciones patrimoniales (Amin 2014). La identidad cultural puede hacer que los individuos que pertenecen a un grupo en una dimensión particular se subdividan a lo largo de otras dimensiones. Por ejemplo, los actores musulmanes en Canadá se identifican como liberales, seculares, progresistas, tradicionales, religiosos y no religiosos (Amin 2014). Este fenómeno complejo juega un papel importante al crear distintos puntos de vista políticos y tendencias de apoyo a las políticas de inmigración, como mostramos más adelante en este estudio.

\section{Etnocentrismo}

El etnocentrismo es el juicio y la comprensión de otras culturas a través del lente de la propia cultura exclusivamente (Omohundro 2008). Fundamentalmente, es una forma de pensar en la que se considera la propia cultura superior a la otra. El etnocentrismo es un concepto que afecta a la mayoría de las personas y se aprende en un nivel inconsciente (Samovar et al. 2013). Se puede reflejar en actividades diarias que son inofensivas, pero también se puede reflejar en problemas más profundos que pueden causar tensiones e incoherencias entre otros grupos de la sociedad. Por lo tanto, el etnocentrismo moldea las opiniones sobre los temas de inmigración. En 


\section{Influencia de la Identidad Cultural en la Percepción Ciudadana - Caso de Estudio}

Vol. 1, núm. 5., (2017)

Blanca Esthela Moscoso; Reema Azar

tiempos políticos y electorales, el etnocentrismo puede direccionar la atención de las preocupaciones económicas a temas de políticas de inmigración (Valentino, Brader, and Jardina 2013). Típicamente, tiende a concentrarse en un grupo específico y en los efectos potenciales que este grupo tiene en la sociedad en general, como en la reactivación económica, la seguridad nacional o el futuro cultural del país (Valentino, Brader, and Jardina 2013; A. J. Banks 2016). Si bien el etnocentrismo no es un fenómeno nuevo, es un fenómeno que ha mostrado una mayor discrepancia entre grupos en los últimos años (Kinder and Kam 2010)

Estas actitudes resultan en expresiones múltiples de xenofobia y, particularmente en el caso de los musulmanes, en una islamofobia, un fenómeno en el que los prejuicios no distinguen entre personas e ideas, creencias, o valores de la cultura islámica (Van der Noll et al. 2017).Algunas de las experiencias típicas de los grupos que están condenados incluyen intimidación, miedo, odio y vergüenza. A menudo, estas emociones crean crisis de identidad (Sadek 2017) y pueden impulsar a personas de un grupo a apoyar políticas en contra de su propio grupo (es decir, políticas de inmigración) (Dunwoody and McFarland 2017)).

\section{La encuesta}

Rasmussen Reports realizó una encuesta de 1,000 "Potenciales votantes de EE. UU." del 4 al 5 de junio de 2017 sobre la opinión pública estadounidense sobre la prohibición de inmigración de Trump (“52\% Say Trump Travel Ban Anti-Terrorist, Not Anti-Muslim” 2017). Según los informes de Rasmussen, la encuesta tiene un error de muestreo de $+/-3 \%$ con un nivel de certeza del $95 \%$. 


\section{Influencia de la Identidad Cultural en la Percepción Ciudadana - Caso de Estudio \\ Vol. 1, núm. 5., (2017) \\ Blanca Esthela Moscoso; Reema Azar}

Este estudio utilizó los datos recopilados en una encuesta de investigación utilizando una metodología de votación automatizada del Centro de informes de Rasmussen. Los sistemas de votación automatizada, a diferencia de un método tradicional asistido por el operador, usan una única voz grabada digitalmente para realizar la entrevista. Esto ayuda a garantizar que a cada encuestado se le presente exactamente la misma encuesta desde el mismo contexto, incluyendo voz, inflexión o acento. Los encuestados se eligieron a través de una selección aleatoria de números telefónicos. El proceso de selección fue estratificado por representación geográfica. Para llegar a aquellos que no usan líneas terrestres, los investigadores crearon una herramienta de encuesta en línea disponible para seleccionar aleatoriamente a los participantes de un panel demográficamente diverso.

Los datos brutos se ponderaron para representar a la población en términos de factores como edad, raza, sexo, partido político y otras categorías. El estudio utilizó las preguntas iniciales proporcionadas por la Oficina del Censo Bureau para determinar posibles votantes. Finalmente, los datos ponderados partidistas consideraron el historial de votación del estado, de las tendencias nacionales y de las encuestas recientes en un estado o área geográfica en particular.

\section{Resultados - análisis estadístico.}

La encuesta telefónica y en línea encontró que el 50\% de los potenciales votantes prefieren prohibir temporalmente la entrada a personas de Siria, Irán, Libia, Somalia, Sudán y Yemen hasta que el gobierno federal mejore sus procedimientos de selección, mientras que el $41 \%$ de los probables votantes se oponen a la prohibición de esta. El 39\% de los probables 


\section{Influencia de la Identidad Cultural en la Percepción Ciudadana - Caso de Estudio}

Vol. 1, núm. 5., (2017)

Blanca Esthela Moscoso; Reema Azar

votantes está de acuerdo en que la prohibición se dirija a los musulmanes para impedir su ingreso al país, mientras que el $52 \%$ dice que la orden está dirigida a evitar "terroristas". Aquellos que creen que la prohibición está dirigida a terroristas, no a musulmanes, argumentan que los siete países de mayoría musulmana afectados por la prohibición, fueron previamente identificados como "paraísos terroristas" por la administración Obama ("52\% Say Trump Travel Ban AntiTerrorist, Not Anti-Muslim” 2017). Las perspectivas de los votantes de EE. UU. con respecto a la prohibición de Trump reflejan las ideologías de los distintos partidos políticos y de distintos grupos demográficos: "Los demócratas son mucho más propensos a considerar la prohibición temporal como antimusulmana-y a oponerse a esta - que los republicanos y los votantes no afiliados " (“52\% Say Trump Travel Ban Anti-Terrorist, Not Anti-Muslim” 2017). Además, el 48\% de los probables votantes menores de 40 se oponen a la prohibición, mientras que la mayoría de los votantes mayores de 40 están a favor ("52\% Say Trump Travel Ban AntiTerrorist, Not Anti-Muslim" 2017).

\section{Discusión.}

Puntos de vista diferentes

Los EE. UU. se dividen en gran medida entre los que apoyan a la Orden Ejecutiva 13769 de Trump y aquellos que se oponen a ella. Algunos a favor de la Orden Ejecutiva pueden hacer afirmaciones extremas de que los refugiados son terroristas, de que los refugiados son musulmanes o de que los refugiados son perjudiciales para la sociedad y la economía ("Seven Common Myths about Refugee Resettlement in the United States" 2016). Otros partidarios encuentran validez en la Orden Ejecutiva con respecto a los recientes ataques terroristas en 


\section{Influencia de la Identidad Cultural en la Percepción Ciudadana - Caso de Estudio}

Vol. 1, núm. 5., (2017)

Blanca Esthela Moscoso; Reema Azar

lugares como Londres y París y en los temores que se manifestaron en declaraciones como la del entonces gobernador Mike Pence "quien respondió a los ataques terroristas en París con el anuncio de que los refugiados de Siria no serían bienvenidos en sus estados "(Kennedy 2015). Adicionalmente y para sorpresa de muchos, incluso los sirio-estadounidenses están divididos en su apoyo u oposición a la Orden Ejecutiva. Fouad Yunis se encuentra entre los sirioestadounidenses que apoyan la prohibición, a pesar de que a su cuñado que reside en Siria se le negó la entrada a los EE. UU. Por ese motivo, Yunis explicó:

Entiendo que [Trump] quiere mantener a Estados Unidos a salvo ... Muchas de estas personas provienen de un área donde no se sabe quiénes son ... Dice que haremos grande a Estados Unidos. Bueno, quiero darle al hombre la oportunidad de hacer que Estados Unidos sea grandioso (The National 2017).

Por otro lado, los votantes en contra de la prohibición citan una serie de razones por las cuales afirman que dicha prohibición no tiene los mejores intereses de la nación. Se han entablado demandas contra Trump, buscando anular la Orden Ejecutiva, y se han escrito varias cartas protestando por sus medidas. Por ejemplo, 80 CEOs, ex CEOs y líderes empresariales que representan a 77 empresas y a 12 industrias firmaron una carta reconociendo "el daño serio a los intereses económicos de EE. UU.—-donde se incluye la potencial pérdida de miles de millones de dólares en pérdidas económicas - que podría resultar de las excesivas restricciones de la política de inmigración sobre preocupaciones de seguridad infundadas "("Forreleasefinalceorefugeesimmigrantsletter1.pdf” n.d.). Además, en lugar de proteger a los EE. UU. del terror, "excluir a los refugiados sirios es exactamente lo que ISIS quiere" (Kennedy 2015). Como 


\section{Influencia de la Identidad Cultural en la Percepción Ciudadana - Caso de Estudio}

Vol. 1, núm. 5., (2017)

Blanca Esthela Moscoso; Reema Azar

señaló S. Kennedy en su publicación (2015), el Centro Nacional de Lucha contra el Terrorismo calculó en el 2011 que los ciudadanos de los EE. UU. "Tienen tantas probabilidades de morir aplastados por sus televisores o muebles cada año como de ser asesinados por terroristas ". Los sentimientos de enojo hacia la prohibición también se derivan de la comprensión de que los EE. UU. es un país que se construyó en base de inmigración, refugiados y religiosos. Muchas de las primeras colonias prometieron libertad religiosa para los puritanos ingleses, y los paralelismos entre los refugiados sirios y los desesperados emigrantes irlandeses de la década de 1840 parecen casi infinitos "(Kruger 2016).

\section{El Rol de la Cultura}

Si bien hay numerosas opiniones a favor y en contra de la prohibición, el hecho es que la cultura ha desempeñado un papel fundamental en la formación de estas perspectivas. Uno de estos aspectos de la cultura de EE. UU., especialmente percibido por quienes se oponen a la prohibición y hacia quienes lo aceptan, es el etnocentrismo. Si bien une a los miembros de una cultura con sentimientos de identidad y pertenencia, el etnocentrismo está vinculado a conceptos de estereotipos, prejuicios y racismo (Samovar et al. 2013), como puede verse en las distintas manifestación del etnocentrismo en xenofobia o "islamofobia" dentro de la esfera pública. Trump provoca este etnocentrismo con sentimientos de nacionalismo y patriotismo en la retórica incluida en su Orden Ejecutiva, incluyendo un lenguaje que evoca la protección de las libertades que los ciudadanos estadounidenses valoran tanto, y del cual otros solo sueñan: "Estados Unidos debe garantizar que los admitidos en este país no adopten actitudes hostiles hacia el país ni hacia 


\section{Influencia de la Identidad Cultural en la Percepción Ciudadana - Caso de Estudio \\ Vol. 1, núm. 5., (2017) \\ Blanca Esthela Moscoso; Reema Azar}

sus principios fundamentales... [La prohibición] garantizará que la admisión de refugiados sirios sea coherente con el interés nacional " (US Executive Order 2017).

Sin embargo, otros aspectos de la cultura de EE. UU. apoyan los argumentos presentados por los votantes contra la prohibición. Por ejemplo, la idea del "sueño americano" representa igualdad de oportunidades para todos, y la Estatua de la Libertad es un icono nacional y "símbolo de libertad, pero también de bienvenida y de gracia" (Kruger 2016). La imagen de los EE. UU. como un "crisol de culturas", y como un país de muchas culturas cuya historia está enraizada en la inmigración de quienes buscan libertades; promueve aún más la frustración y la inaceptabilidad del Orden Ejecutivo. La historia de EE. UU. está llena de simbolismos e íconos culturales que indican que el país debería ser un lugar de bienvenida, y no uno que rechaza culturalmente a los demás.

\section{Conclusiones.}

Como se ha visto en eventos recientes, los ciudadanos estadounidenses se dividen en gran parte entre defensores y críticos de la Orden Ejecutiva 13769 del presidente Trump, también llamada "prohibición musulmana". Hay al menos dos correlaciones en términos de quién podría apoyar o criticar la prohibición: es más probable que los que apoyan la prohibición se consideren republicanos y tengan más de 40 años, mientras que aquellos que condenan la prohibición podrían estar afiliados como demócratas y estar entre los 18 y 40 años. Se requiere más investigación sobre cuáles son las características culturales dominantes que existen—si las haydentro de cada categoría de afiliación y edad de cada partido. Sin embargo, si bien no se puede 


\section{Influencia de la Identidad Cultural en la Percepción Ciudadana - Caso de Estudio}

Vol. 1, núm. 5., (2017)

Blanca Esthela Moscoso; Reema Azar

negar que los votantes estadounidenses están divididos sobre el tema, quizás lo más intrigante es que incluso los ciudadanos sirio-estadounidenses difieren en sus puntos de vista; ya que no todos ven la Orden Ejecutiva de Trump como racista o perjudicial para la sociedad. Una comparación de las identidades culturales de los sirios-estadounidenses con los sirios revelará las dimensiones a lo largo de las cuales estos dos grupos difieren, y ofrecerá una explicación de por qué algunos sirios-estadounidenses apoyan la prohibición de la inmigración. La cultura de EE. UU. juega un papel crucial en el desarrollo de la polarización de sentimientos hacia la prohibición. Por un lado, el etnocentrismo se hace evidente en la esfera pública, como lo ha sido desde la colonización de América. Sin embargo, la cultura estadounidense es rica en símbolos de bienvenida e inclusividad, como ejemplifica la Estatua de la Libertad, que se para con una antorcha para iluminar el camino hacia la Tierra de los Libres. La prohibición de los refugiados es un tema crítico que sin duda tendrá implicaciones futuras sobre cómo se identifica a los EE. UU. tanto a nivel nacional como internacional. Con una ciudadanía dividida y una identidad cultural chocante, uno debe preguntarse: ¿Qué representa realmente Estados Unidos?

\section{Referencias.}

“52\% Say Trump Travel Ban Anti-Terrorist, Not Anti-Muslim.” 2017. Rasmussen Reports. http://www.rasmussenreports.com/public_content/politics/current_events/israel_the_mid dle_east/52_say_trump_travel_ban_anti_terrorist_not_anti_muslim.

Amin, Sara N. 2014. "The Impact of Identity Politics in Challenging National Narratives: A Case Study among Canadian Muslims." Studies in Ethnicity and Nationalism 14 (3):418-435.

Banks, Antoine. 2017. "Anger Makes Ethnocentrism among Whites a Stronger Predictor of Racial and Immigration Policy Opinions." USApp-American Politics and Policy Blog. 


\section{Influencia de la Identidad Cultural en la Percepción Ciudadana - Caso de Estudio}

Vol. 1, núm. 5., (2017)

Blanca Esthela Moscoso; Reema Azar

Banks, Antoine J. 2016. “Are Group Cues Necessary? How Anger Makes Ethnocentrism among Whites a Stronger Predictor of Racial and Immigration Policy Opinions." Political Behavior 38 (3):635-657.

Claassen, Christopher. 2016. "Group Entitlement, Anger and Participation in Intergroup Violence.” British Journal of Political Science 46 (1):127-148.

Dunwoody, Philip T., and Sam G. McFarland. 2017. "Support for Anti-Muslim Policies: The Role of Political Traits and Threat Perception." Political Psychology.

"Forrelease-finalceorefugeesimmigrantsletter1.pdf." n.d. Accessed December 11, 2017. https://www.rescue.org/sites/default/files/document/1354/forreleasefinalceorefugeesimmigrantsletter1.pdf.

Hogg, Michael A. 2016. "Social Identity Theory." In Understanding Peace and Conflict Through Social Identity Theory, 3-17. Springer.

Kennedy, Sheila Suess. 2015. "What's Really Driving Syrian Refugee Ban." Indianapolis Business Journal, November, 15.

Kinder, Donald R., and Cindy D. Kam. 2010. Us against Them: Ethnocentric Foundations of American Opinion. University of Chicago Press.

King, Colin. 2004. "Race and Cultural Identity: Playing the Race Game inside Football." Leisure Studies 23 (1):19-30.

Kruger, Kyra. 2016. "A Misperceived America: The Syrian Refugee Crisis Is an Issue beyond Bipartisan Politics." University Wire; Carlsbad, October 18, 2016, sec. Opinion. https://search.proquest.com/docview/1830473870/abstract/F0C5585069864984PQ/1.

Mackie, Diane M., Thierry Devos, and Eliot R. Smith. 2000. "Intergroup Emotions: Explaining Offensive Action Tendencies in an Intergroup Context." Journal of Personality and Social Psychology 79 (4):602.

Omohundro, John T. 2008. Thinking like an Anthropologist: A Practical Introduction to Cultural Anthropology. McGraw Hill.

Roccas, Sonia, and Marilynn B. Brewer. 2002. "Social Identity Complexity." Personality and Social Psychology Review 6 (2):88-106.

Sadek, Noha. 2017. "Islamophobia, Shame, and the Collapse of Muslim Identities." International Journal of Applied Psychoanalytic Studies 14 (3):200-221.

Samovar, L.A., R.E. Porter, E.R. McDaniel, and C.S. Roy. 2013. Communication Between Cultures. 8th ed. Boston, MA: Wadsworth, Cengage Learning.

Revista Científica Mundo de la Investigación y el Conocimiento. 1(5). pp. 799-813 


\section{Influencia de la Identidad Cultural en la Percepción Ciudadana - Caso de Estudio}

Vol. 1, núm. 5., (2017)

Blanca Esthela Moscoso; Reema Azar

"Seven Common Myths about Refugee Resettlement in the United States." 2016. International Rescue Committee (IRC). September 19, 2016. https://www.rescue.org/article/sevencommon-myths-about-refugee-resettlement-united-states.

Shiv, Baba, George Loewenstein, Antoine Bechara, Hanna Damasio, and Antonio R Damasio. 2005. "Investment Behavior and the Negative Side of Emotion." Psychological Science 16 (6):435-39. https://doi.org/10.1111/j.0956-7976.2005.01553.x.

The National. 2017. Syrian Americans on Trump's Travel Ban. https://www.youtube.com/watch?v=9NkNVb4lWu0.

US Executive Order. 2017. "Executive Order: Protecting the Nation from Foreign Terrorist Entry into the United States."

Valentino, Nicholas A., Ted Brader, and Ashley E. Jardina. 2013. "Immigration Opposition among US Whites: General Ethnocentrism or Media Priming of Attitudes about Latinos?" Political Psychology 34 (2):149-166.

Van der Noll, Jolanda, Vassilis Saroglou, David Latour, and Nathalie Dolezal. 2017. "Western Anti-Muslim Prejudice: Value Conflict or Discrimination of Persons Too?" Political Psychology. 